Check for updates

Cite this: RSC Adv., 2018, 8, 23847

\title{
SnS/C nanocomposites for high-performance sodium ion battery anodes $\dagger$
}

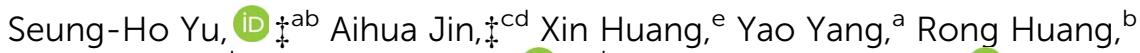 \\ Joel D. Brock, ${ }^{\text {be }}$ Yung-Eun Sung $\mathbb{D}^{* c d}$ and Héctor D. Abruña (iD *a
}

Sodium-ion batteries have been considered as one of the most promising types of batteries, beyond lithium-ion batteries, for large-scale energy storage applications. However, their deployment hinges on the development of new anode materials, since it has been shown that many important anode materials employed in lithium ion batteries, such as graphite and silicon, are inadequate for sodium-ion batteries. We have simply prepared novel SnS/C nanocomposites through a top-down approach as anode materials for sodium-ion batteries. Their electrochemical performance has been significantly improved when compared to bare SnS, especially in terms of cycling stability and rate capabilities. SnS/C nanocomposites exhibit excellent capacity retention, at various current rates, and deliver capacities as high as $400 \mathrm{~mA} \mathrm{~h} \mathrm{~g}^{-1}$ even at the high current density of $800 \mathrm{~mA} \mathrm{~g}^{-1}$ (2C). Ex situ transmission electron microscopy, X-ray diffraction and operando X-ray absorption near edge structure studies have been performed in order to unravel the reaction mechanism of the SnS/C nanocomposites.

Received 24th May 2018

Accepted 21st June 2018

DOI: $10.1039 / \mathrm{c} 8 \mathrm{ra04421 \textrm {j }}$

rsc.li/rsc-advances

has been intensively studied for LIB anodes, due to its exceptionally high theoretical capacity, the redox potential of silicon is too low for application in SIBs. ${ }^{8,14}$ Instead, other alloy-type materials such as Sn-, Sb-, and Ge-based materials have been examined in detail as high-capacity anodes for SIBs. ${ }^{15-25}$ In addition, it has been reported that various transition metal oxides, sulfides and selenides can store sodium ions reversibly through intercalation and/or conversion reactions. ${ }^{26-37}$ Furthermore, several approaches have been introduced to facilitate intercalation of sodium ions into graphite by using expanded graphite, and ether-based electrolytes. ${ }^{11,38-40}$ However, these materials still have limitations such as low capacity, poor rate capability, rapid capacity fade and complex preparation process.

Herein, we report a simple preparation method for SnS/C nanocomposites, and their application to sodium-ion battery anodes. The grain size of $\mathrm{SnS}$ could reach about $10 \mathrm{~nm}$, and $\mathrm{SnS}$ particles were well surrounded by carbon particles thereby increasing conductivity. The nanocomposites exhibited significantly enhanced electrochemical performance when compared to those of bare SnS electrodes. The nanocomposites delivered a reversible capacity of $480.0 \mathrm{~mA} \mathrm{~h} \mathrm{~g}{ }^{-1}$ with negligible capacity fade during 50 cycles at a current density of $100 \mathrm{~mA} \mathrm{~g}^{-1}$. The composites also showed outstanding rate capabilities, delivering $\sim 93 \%$ of their capacity when the current density was increased by a factor of ten (from 50 to $500 \mathrm{~mA} \mathrm{~g}^{-1}$ ). Furthermore, the reaction mechanism of SnS/C nanocomposites was studied using ex situ transmission electron microscopy (TEM), $\mathrm{X}$-ray diffraction (XRD) and operando X-ray absorption near edge structure (XANES).

\footnotetext{
${ }^{a}$ Department of Chemistry and Chemical Biology, Cornell University, Ithaca, New York 14853, USA. E-mail: hda1@cornell.edu

Cornell High Energy Synchrotron Source, Cornell University, Ithaca, NY 14853 Republic of Korea

${ }^{d}$ School of Chemical and Biological Engineering, Seoul National University, Seoul 151742, Republic of Korea. E-mail: ysung@snu.ac.kr

${ }^{e}$ School of Applied and Engineering Physics, Cornell University, Ithaca, New York 14853, USA

$\dagger$ Electronic supplementary information (ESI) available. See DOI: 10.1039/c8ra04421j

\$ These authors contributed equally to this work.
} 


\section{Experimental}

\section{Preparation of SnS/C nanocomposites}

Tin(II) sulfide (Aldrich) and super P carbon were mixed in a weight ratio of $7: 3$, and the mixture was transferred to a ball-milling container in an argon-filled glove box. Mechanical ball-milling was conducted with a PULVERISETTE7 (Fritsch) at a speed of $500 \mathrm{rpm}$ for $10 \mathrm{~h}$.

\section{Characterizations}

Field-emission scanning electron microscopy (FE-SEM) analysis was conducted with a SUPRA 55VP (Carl Zeiss). X-ray diffraction patterns were obtained using a Bruker D-5005 with $\mathrm{Cu} \mathrm{K} \alpha$ radiation. Operando XANES experiments were conducted at the F-3 station of the Cornell High Energy Synchrotron Source (CHESS). High-resolution transmission electron microscopy (HRTEM) images, and corresponding selected area electron diffraction (SAED), were obtained using an FEI field-emissiongun (FEG) Tecnai F-20 operated at $200 \mathrm{kV}$.

\section{Electrochemical characterization}

A slurry, composed of $70 \mathrm{wt} \%$ of active material, $15 \mathrm{wt} \%$ of super $\mathrm{P}$ carbon and $15 \mathrm{wt} \%$ of poly(acrylic acid) in $\mathrm{N}$-methyl-2pyrrolidinone solvent, was coated on the current collector. The electrodes were subsequently vacuum dried in an oven overnight at $120{ }^{\circ} \mathrm{C}$. The loading mass of active material on electrode is about $1.4 \mathrm{mg} \mathrm{cm} \mathrm{cm}^{-2}$. The coin cells (CR-2032) were assembled in an argon-filled glove box with sodium metal as both counter and reference electrodes. A glass fiber filter (GF/C, Whatman) was used as separator. $1.0 \mathrm{M} \mathrm{NaClO}_{4}$ in ethylene carbonate (EC)/propylene carbonate (PC) (1:1 by volume) with $5 \mathrm{wt} \%$ fluoroethylene carbonate (FEC) as additive was used as the electrolyte. Coin cells were tested on BT-2000 (Arbin Instruments) and WBCS3000 cyclers (WanA Tech) over a voltage range from $0.01 \mathrm{~V}$ to $3.0 \mathrm{~V}\left(v s . \mathrm{Na}^{+} / \mathrm{Na}\right)$.

\section{Results and discussion}

\section{Characterization of prepared SnS/C nanocomposites}

Fig. 1 presents X-ray diffraction (XRD) patterns of bare commercial $\mathrm{SnS}$ and SnS/C nanocomposites. The XRD peaks of bare SnS powder are well matched to values of the $\mathrm{SnS}$ reference card (JCPDS no. 14-0620, orthorhombic structure, $a=4.33, b=11.19$ and $c=3.98)$. The $(040)$ peak of bare $\mathrm{SnS}$ is overwhelmingly stronger when compared to other peaks, indicating that the SnS layers are preferentially stacked in the [010] direction (see crystal structure in inset of Fig. 1). The XRD peaks of the SnS/C nanocomposites are much broader than those of bare $\mathrm{SnS}$, indicating that the grain size is much smaller. The calculated grain size of $\mathrm{SnS} / \mathrm{C}$ nanocomposites, by the Scherrer equation is about $10 \mathrm{~nm}$. It is worthwhile to note that the intensities of the (040) and (080) peaks decreased more significantly than other peaks after ballmilling. This suggests that the layers of SnS are more easily broken in the [010] direction than others during ball-milling (see Fig. 2a and b). It is worthwhile to note that obtaining nanosized material ( $<100 \mathrm{~nm})$ using ball-milling is difficult in general, but
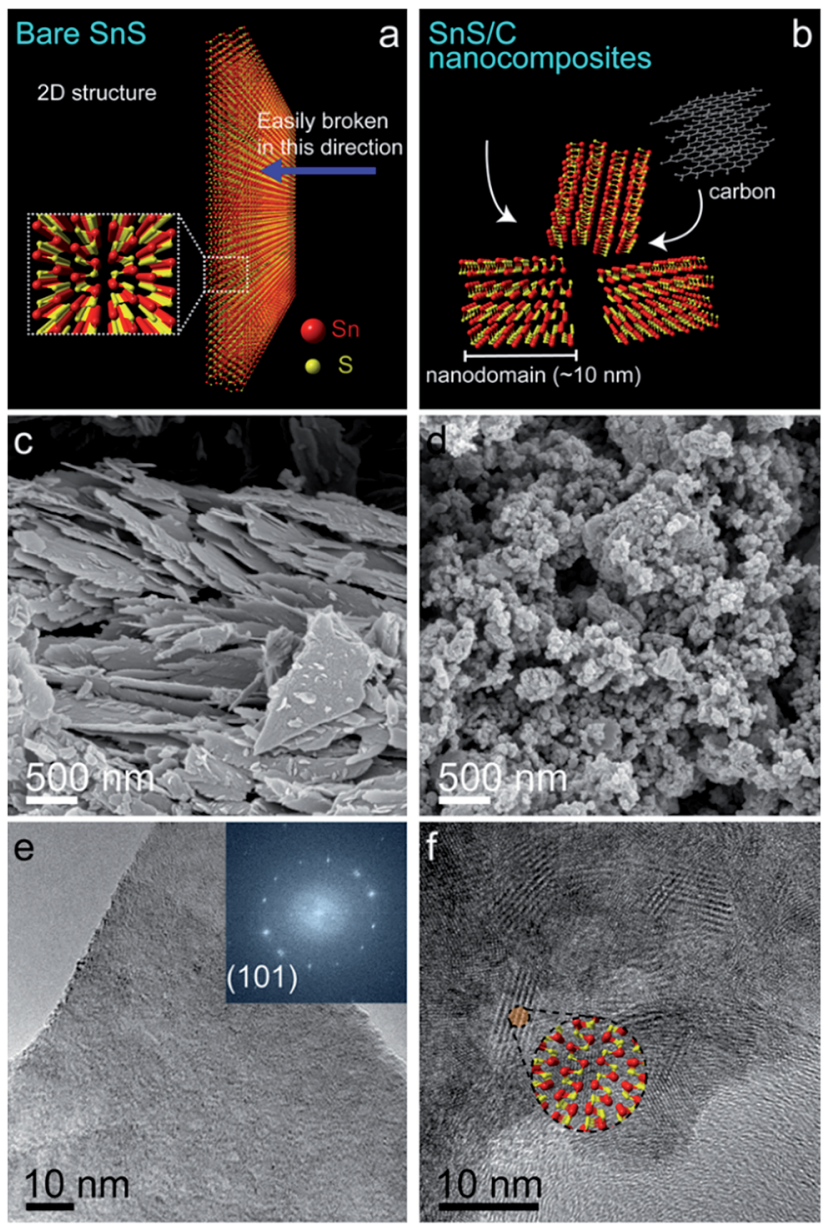

Fig. 1 Schematic illustration of morphology of (a) bare SnS and (b) SnS/C nanocomposites. (c) SEM image and (e) TEM images of bare SnS. Inset of (e) shows a fast Fourier transform (FFT) image. (d) SEM image and (f) TEM images of SnS/C nanocomposites.

intrinsically bare SnS has two-dimensional character with preferential direction, and thickness in the other direction is small. Therefore, small-sized SnS particles can be obtained simply using

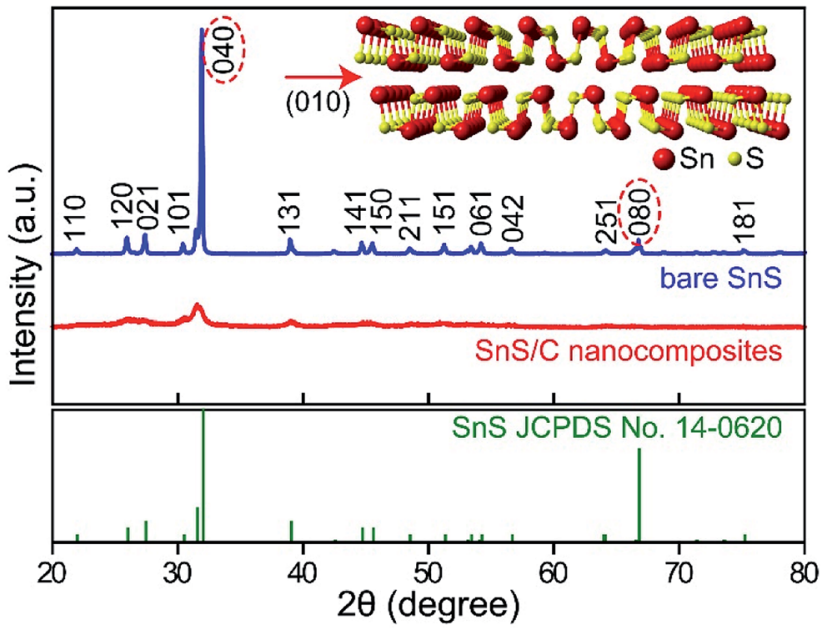

Fig. 2 XRD patterns of bare commercial SnS and SnS/C nanocomposites (top panel) and SnS from JCPDS no. 14-0620 (bottom panel). Inset shows crystal structure of SnS. 
ball-milling, even size can reach to about $10 \mathrm{~nm}$. XANES spectrum of $\mathrm{SnS} / \mathrm{C}$ nanocomposites is overlapped with that of bare SnS, indicating that no phase transition (reduction/oxidation) was occurred during ball-milling process (see Fig. S1 in ESI $\dagger$ ).

In scanning electron microscopy (SEM) and transmission electron microscopy (TEM) images of bare SnS (Fig. 2c and e), several thin $(\sim 50 \mathrm{~nm})$ micron-sized SnS layers are observed. Based on the FFT (inset to Fig. 2e), the most prominently observed interplanar distance is $0.29 \mathrm{~nm}$ for the (101) planes, supporting our earlier statement that the layers are preferentially oriented along the [010] direction. In the SnS/C nanocomposites, the SnS particles have a nonhomogeneous size distribution, and they are well covered by carbon particles (Fig. 2d and f). In the HRTEM and STEM images of SnS/C nanocomposites (Fig. 2f, S2 and Fig S3†), it can be observed that they still have 2D characteristic, and the typical grain size is $\sim 10 \mathrm{~nm}$, which is in good agreement with value calculated from the XRD results presented above.

\section{Electrochemical properties of SnS/C nanocomposites} electrochemical characterization

The electrochemical properties of the bare $\mathrm{SnS}$ and $\mathrm{SnS} / \mathrm{C}$ nanocomposites were evaluated as anode materials for SIBs. The cells were galvanostatically discharged and charged at a current density of $100 \mathrm{~mA} \mathrm{~g}^{-1}$ over the voltage range of $0.01-$ $3.0 \mathrm{~V} \mathrm{(vs.} \mathrm{Na}^{+} / \mathrm{Na}$ ) (Fig. 3). For the bare $\mathrm{SnS}$, the initial sodiation and desodiation capacities were 740.2 and $592.5 \mathrm{~mA} \mathrm{~h} \mathrm{~g}{ }^{-1}$, respectively (Fig. 3a). During the initial sodiation process a large plateau was observed at about $0.85 \mathrm{~V}$. However, the plateau moved to higher voltages in subsequent cycles. Similarly, in the cyclic voltammetric responses, an intense peak was observed in the first reduction cycle. However, in subsequent cycles, this peak disappeared and was replaced by two separate peaks at higher potentials (0.97 and $0.67 \mathrm{~V}$ ) (Fig. 3b). Such activation processes during the initial discharge are typically observed in conversion reaction-based materials in LIBs and SIBs and often result in
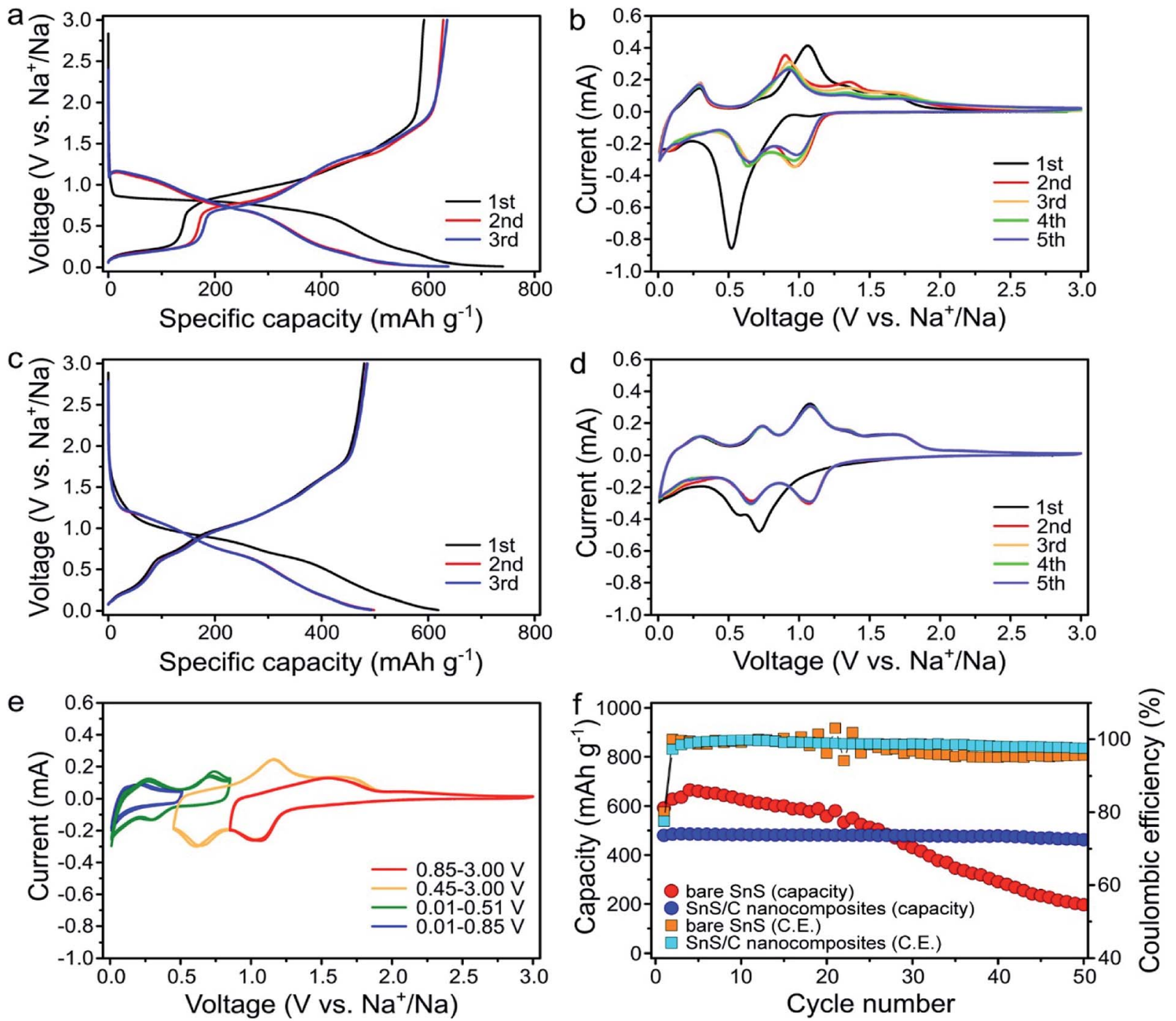

Fig. 3 (a) First three voltage profiles of bare $\mathrm{SnS}$ at a current density of $100 \mathrm{~mA} \mathrm{~g}^{-1}$. (b) Cyclic voltammograms of bare SnS at a scan rate of $0.1 \mathrm{mV}$ $\mathrm{s}^{-1}$. (c) First three voltage profiles of SnS/C nanocomposites at a current density of $100 \mathrm{~mA} \mathrm{~g}^{-1}$. (d) Cyclic voltammograms of SnS/C nanocomposites at a scan rate of $0.1 \mathrm{mV} \mathrm{s}^{-1}$ between 0.01 and $3.0 \mathrm{~V}$ (e) cyclic voltammograms of SnS/C nanocomposites at a scan rate of $0.1 \mathrm{mV} \mathrm{s}{ }^{-1}$ with different cut off voltage ranges. (f) Cycle performance of bare SnS and SnS/C nanocomposites at a current density of $100 \mathrm{~mA} \mathrm{~g}^{-1}$. 
a dramatic decrease in the domain size..$^{41-43}$ Fig. $3 \mathrm{c}$ and $\mathrm{d}$ present discharge/charge profiles and cyclic voltammograms (CVs) for SnS/C nanocomposites, respectively. While in Fig. 3c, the first and second sodiation profiles are still different, the differences are much smaller when compared to the first two profiles for bare SnS shown in Fig. 3a. The grain size of the initial SnS/C nanocomposites is much smaller $(\sim 10 \mathrm{~nm})$ than that of the bare $\mathrm{SnS}$, so the differences, before and after the first cycle in SnS/C nanocomposites are less significant. In Fig. 3d, many peaks are observed during the reduction and oxidation processes in SnS/C nanocomposites. In an effort to match reduction and anodic peaks, we performed cyclic voltammetry with different cut-off voltages after full sodiation (Fig. 3e). Based on these results, we feel confident in correcting the various cathodic peaks at 1.08, $0.65,0.30$, and $0.01 \mathrm{~V}$ and anodic peaks at $1.70,1.07,0.74$, and 0.28 , as representing redox pairs, respectively. It is also worth making some additional observations regarding the voltammetric behavior. First, the CVs of $\mathrm{SnS} / \mathrm{C}$ nanocomposites well overlapped (Fig. 3d), indicating that stability of $\mathrm{SnS} / \mathrm{C}$ nanocomposites was enhanced. Secondly, the peaks in the SnS/C nanocomposites are much better defined and with a lower overpotential than those of bare $\mathrm{SnS}$, indicating improved reaction kinetics in the SnS/C composites. Fig. $3 \mathrm{f}$ presents the cycling performance of bare $\mathrm{SnS}$ and $\mathrm{SnS} / \mathrm{C}$ nanocomposites. The initial desodiation capacity of SnS/C nanocomposites was $480.0 \mathrm{~mA} \mathrm{~h} \mathrm{~g}{ }^{-1}$, which translates to $685.7 \mathrm{~mA} \mathrm{~h} \mathrm{~g}^{-1}$ when calculated based only on the weight of SnS. While the capacity of the bare SnS electrode decreased continuously during the first 50 cycles, the capacity retention of $\mathrm{SnS} / \mathrm{C}$ nanocomposites was dramatically higher, reaching $96.3 \%$ after 50 cycles.
Rate properties of bare $\mathrm{SnS}$ and $\mathrm{SnS} / \mathrm{C}$ nanocomposites were evaluated by varying the current density stepwise from 50 to $800 \mathrm{~mA} \mathrm{~g}^{-1}$ (Fig. 4a). Upon increasing the current density, the capacity of bare $\mathrm{SnS}$ decreased significantly. At the high current density of $800 \mathrm{~mA} \mathrm{~g}^{-1}$, bare $\mathrm{SnS}$ delivered a low capacity $\left(<200 \mathrm{~mA} \mathrm{~h} \mathrm{~g}^{-1}\right)$, while SnS/C nanocomposites delivered a much higher capacity of about $400 \mathrm{~mA} \mathrm{~h} \mathrm{~g}^{-1}$. In addition, SnS/C composites exhibited stable cycling even at a current density of $800 \mathrm{~mA} \mathrm{~g}^{-1}$. This is especially important when one considers that this current density enables two full sodiation or desodiation processess in one hour. When the current density was changed back to $50 \mathrm{~mA} \mathrm{~g}^{-1}$, after charging/discharging at $800 \mathrm{~mA} \mathrm{~g}^{-1}$, the capacity of SnS/C nanocomposites recovered their initial value, indicating that there was no structural damage to the material during high rate cycling. The voltage profiles for the fifth cycle of each current step are plotted in Fig. $4 \mathrm{~b}$ and $\mathrm{c}$ for bare $\mathrm{SnS}$ and $\mathrm{SnS} /$ C nanocomposites, respectively. For bare SnS (Fig. 4b), the voltage profiles exhibited large overpotentials and the reaction plateaus were ill-defined at high current densities. Contrary to that, the $\mathrm{SnS} /$ C composites exhibited much smaller overpotentials in the voltage profiles, and the reaction plateaus were also clearly defined even at a current density of $800 \mathrm{~mA} \mathrm{~g}^{-1}$. Fig. $4 \mathrm{~d}$ shows the cycle performance of SnS/C nanocomposites at various current densities (see Fig. $\mathrm{S} 4 \uparrow$ for cycle performance of ball-milled $\mathrm{SnS}$ without carbon at $500 \mathrm{~mA} \mathrm{~g}^{-1}$ ). The first reversible capacities at current densities of 50 and $500 \mathrm{~mA} \mathrm{~g}^{-1}$ were 488.2 and $441.2 \mathrm{~mA} \mathrm{~h} \mathrm{~g}^{-1}$, indicating a capacity retention of over $90 \%$ even after a tenfold increase in current density. In addition, $\mathrm{SnS} / \mathrm{C}$ nanocomposites exhibited excellent cycling stability during 50 cycles at all current densities from 50 to $500 \mathrm{~mA} \mathrm{~g}^{-1}$. In particular, the capacity retention was
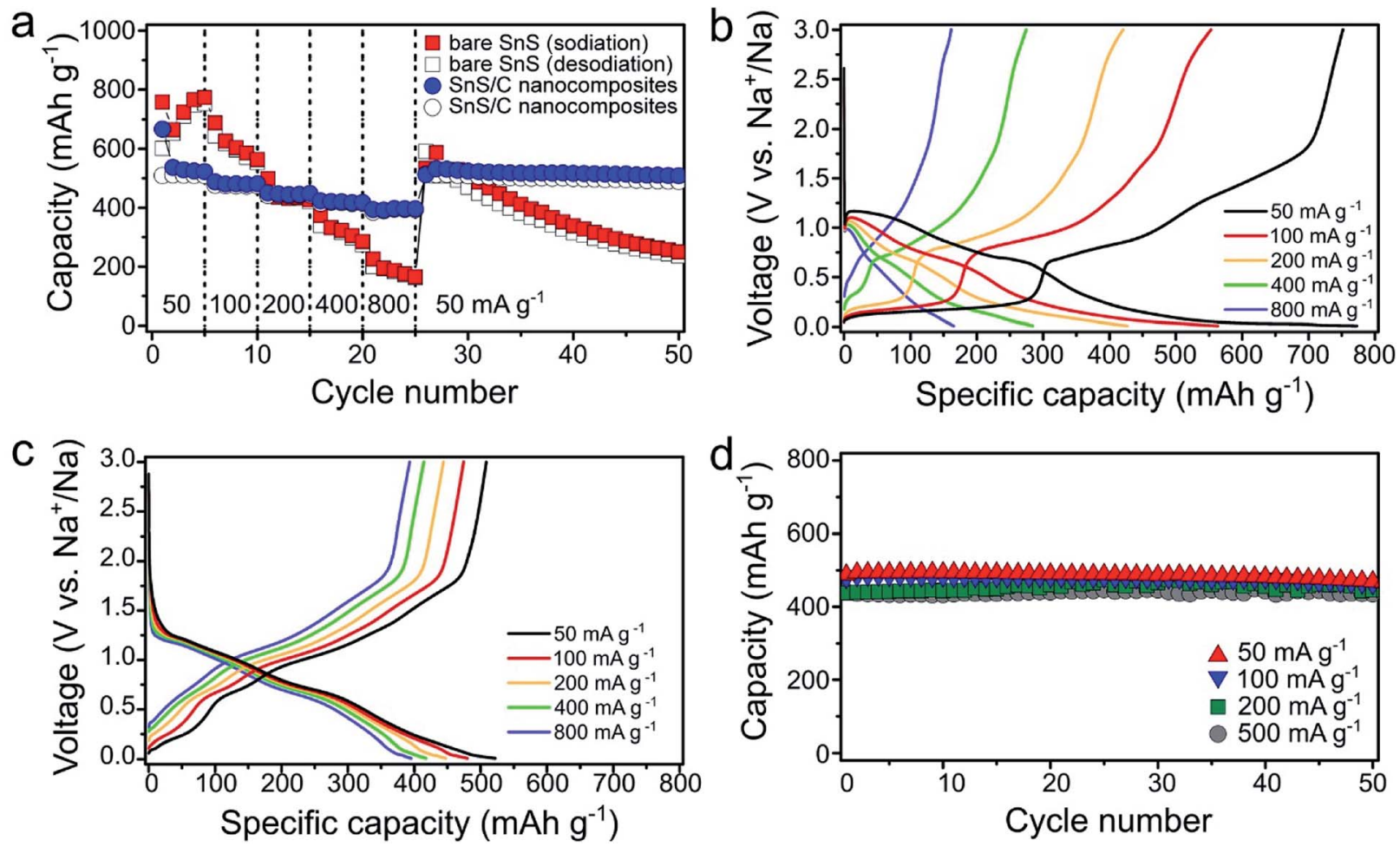

Fig. 4 (a) Rate properties of bare SnS and SnS/C nanocomposites. Voltage profiles of (b) bare SnS and (c) SnS/C nanocomposites at various current densities. (d) Cycle performance of SnS/C nanocomposites at various current densities. 
$98.7 \%$ at $500 \mathrm{~mA} \mathrm{~g}^{-1}$; a value that is much higher than those of other reported anode materials. ${ }^{44-47}$ We also prepared SnS/C nanocomposites using mesoporous carbon (CMK), instead of super $\mathrm{P}$ carbon, and these exhibited much improved cycling stability and rate performance (Fig. S5 $\dagger$ ). This confirms that SnS can exhibit excellent electrochemical properties when it is ballmilled with carbon, with virtually no dependence on the type of carbon employed, so that $\mathrm{SnS} / \mathrm{C}$ nanocomposites are very attractive not only in terms of their battery performance but also in practical terms.

\section{Reaction mechanism of SnS/C nanocomposites}

In order to better understand the reaction mechanism of the SnS/C nanocomposites, ex situ XRD and operando X-ray
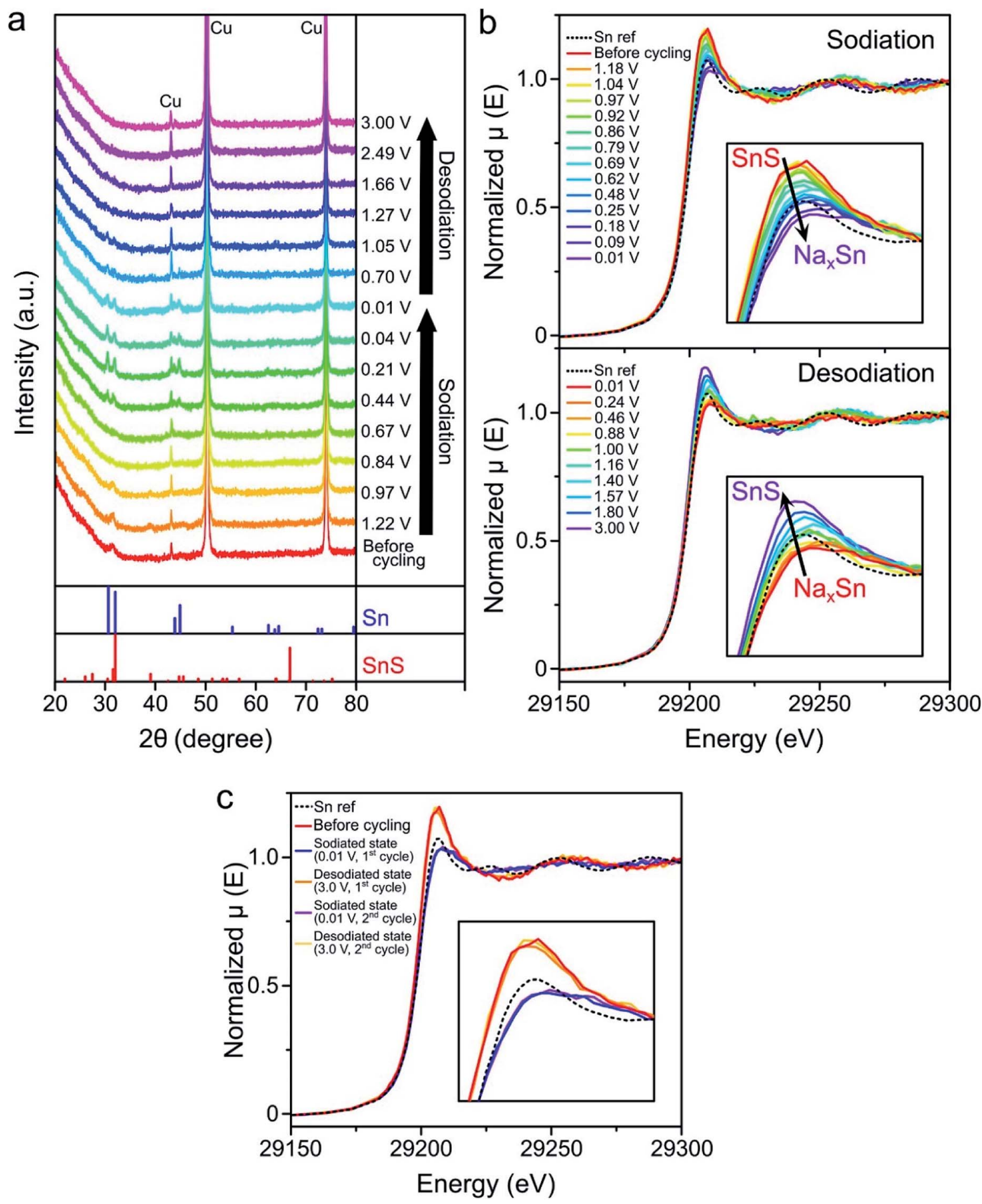

Fig. 5 (a) Ex situ XRD patterns and (b) operando XANES spectra of SnS/C nanocomposites at the Sn K edge during the first sodiation and desodiation process. (c) XANES spectra of SnS/C nanocomposites at the Sn K edge before battery testing, and after the first and second sodiation and desodiation processes. 
absorption near edge structure (XANES) analyses were performed (Fig. 5). Both $\mathrm{Al}$ and $\mathrm{Cu}$ can be used as current collectors for SIB anodes, because $\mathrm{Al}$ does not react with $\mathrm{Na}$ ions. $\mathrm{Al}$ is less expensive than $\mathrm{Cu}$, so $\mathrm{Al}$ is more frequently used for SIB anodes. However, since the main XRD peaks of $\mathrm{SnS}$ is overlapped with $\mathrm{Al}$ $\mathrm{XRD}$ peaks, $\mathrm{Cu}$ foil was used as a current collector for XRD experiment. The broad XRD peak from SnS started to weaken during the initial sodiation, and disappeared at $0.67 \mathrm{~V}$. At the same time, the peaks ascribed to Sn metal appeared, indicating that the conversion reaction of SnS occurred to form Sn metal clusters and amorphous sodium sulfide. At the fully sodiated state $(0.01 \mathrm{~V})$, Sn metal peaks can be seen in the XRD patterns. Additionally, weak electron diffraction patterns, corresponding to $\mathrm{Sn}$ metal and the $\mathrm{Na}_{15} \mathrm{Sn}_{4}$ phase were observed in the SAED pattern at the fully sodiated state $(0.01 \mathrm{~V})$ (Fig. S6b $\dagger)$. This suggests that some of the $\mathrm{Sn}$ metal clusters reacted with sodium to form $\mathrm{Na}_{15} \mathrm{Sn}_{4}$ through alloy reactions, but it was difficult to detect diffraction patterns of $\mathrm{Na}-\mathrm{Sn}$ alloy phases likely because of their small particle size and low crystallinity. ${ }^{48,49}$ During desodiation, XRD peaks from Sn totally disappeared, and no other peaks nor patterns, from active material, were observed at the fully desodiated state $(3.0 \mathrm{~V})$. Similarly, only carbon electron diffraction patterns were observed at the fully desodiated state (Fig. S6d $\dagger$ ). In the Sn XANES spectra, the peak height continuously decreased during sodiation (Fig. 5b), reached an intensity comparable to that of the $\mathrm{Sn}$ reference metal at $0.48 \mathrm{~V}$, and further decreased until fully discharged. This indicates that the conversion reaction of $\mathrm{SnS}$ to $\mathrm{Sn}$ metal was complete at around $0.5 \mathrm{~V}$ and that further sodiation processes into $\mathrm{Sn}$ metal occurred at lower potentials. During desodiation (Fig. 5b), the peak height continuously increased, reached an intensity comparable to that of the Sn reference metal at about $1.00 \mathrm{~V}$, and kept increasing further, indicating that the $\mathrm{Na}-\mathrm{Sn}$ alloy oxidized to Sn metal, and continuously changed to SnS above $1.0 \mathrm{~V}$. It is worth noting that the XANES spectra of the SnS/C nanocomposites before testing, and after the first and second cycles, at the charged state, totally overlapped, indicating that the reactions of $\mathrm{SnS} / \mathrm{C}$ nanocomposites are fully reversible although the reactions go through multiple steps involving conversion and alloying reactions. When compared to tin oxidebased materials, which also go through both conversion and alloying reactions similarly to tin sulfide, their conversion reactions are known to be irreversible..$^{50,51}$

\section{Conclusions}

SnS/C nanocomposites were obtained through a practical and economical top-down approach. The unique structural properties of $\mathrm{SnS}$ enable to reach small nanodomain of about $10 \mathrm{~nm}$ using ball-milling process. The $\mathrm{SnS} / \mathrm{C}$ nanocomposites exhibited superior electrochemical performance, compared to bare $\mathrm{SnS}$, for sodium ion batteries. The $\mathrm{SnS} / \mathrm{C}$ nanocomposites delivered a high desodiation capacity of about $480 \mathrm{~mA} \mathrm{~h} \mathrm{~g}$ (about $686 \mathrm{~mA} \mathrm{~h} \mathrm{~g}^{-1}$ when calculated based on the weight of $\mathrm{SnS}$ alone) at a current density of $100 \mathrm{~mA} \mathrm{~h} \mathrm{~g}^{-1}$ through multiple reaction steps including conversion and alloying reactions. They also showed excellent cycling stability at various current densities, with more than $90 \%$ of its capacity delivered when the current increased from 50 to $500 \mathrm{~mA} \mathrm{~g}^{-1}$. The sodiation/ desodiation reaction mechanism of $\mathrm{SnS} / \mathrm{C}$ composites was studied by ex situ TEM, XRD and operando XANES analyses. The reactions of $\mathrm{SnS} / \mathrm{C}$ nanocomposites were remarkably reversible, as established by cyclic voltammetry and operando XANES analysis. We believe that the approach that we have used in this study, for the preparation of SnS/C nanocomposites, will provide valuable insights into developing practical and promising anode materials for sodium-ion batteries.

\section{Conflicts of interest}

There are no conflicts to declare.

\section{Acknowledgements}

This work is based upon research conducted at the Cornell High Energy Synchrotron Source (CHESS) which is supported by the National Science Foundation and the National Institutes of Health/National Institute of General Medical Sciences under NSF award DMR-1332208. S.-H. Yu acknowledges support from CHESS and the Energy Materials Center at Cornell (emc2). Y.-E. Sung acknowledges the financial support by IBS-R006-A2.

\section{Notes and references}

1 M. D. Slater, D. Kim, E. Lee and C. S. Johnson, Adv. Funct. Mater., 2013, 23, 947.

2 H. Pan, Y.-S. Hu and L. Chen, Energy Environ. Sci., 2013, 6, 2338.

3 N. Yabuuchi, K. Kubota, M. Dahbi and S. Komaba, Chem. Rev., 2014, 114, 11636.

4 S.-W. Kim, D.-H. Seo, X. Ma, G. Ceder and K. Kang, Adv. Energy Mater., 2012, 2, 710.

5 Y. Wang, X. Yu, S. Xu, J. Bai, R. Xiao, Y.-S. Hu, H. Li, X.-Q. Yang, L. Chen and X. Huang, Nat. Commun., 2013, 4(2365), 6.

6 J.-Y. Hwang, S.-T. Myung and Y.-K. Sun, Chem. Soc. Rev., 2017, 46, 3529.

7 K. Kubota and S. Komaba, J. Electrochem. Soc., 2015, 162, A2538.

8 Y. Kim, K.-H. Ha, S. M. Oh and K. T. Lee, Chem.-Eur. J., 2014, 20, 11980.

9 J. Qian, X. Wu, Y. Cao, X. Ai and H. Yang, Angew. Chem., Int. Ed., 2013, 125, 4731.

10 M. Dahbi, N. Yabuuchi, K. Kubota, K. Tokiwa and S. Komaba, Phys. Chem. Chem. Phys., 2014, 16, 15007.

11 Y. Wen, K. He, Y. Zhu, F. Han, Y. Xu, I. Matsuda, Y. Ishii, J. Cumings and C. Wang, Nat. Commun., 2014, 5, 4033.

12 S. Komaba, W. Murata, T. Ishikawa, N. Yabuuchi, T. Ozeki, T. Nakayama, A. Ogata, K. Gotoh and K. Fujiwara, Adv. Funct. Mater., 2011, 21, 3859.

13 E. Irisarri, A. Ponrouch and M. Palacin, J. Electrochem. Soc., 2015, 162, A2476.

14 V. Chevrier and G. Ceder, J. Electrochem. Soc., 2011, 158, A1011. 
15 L. D. Ellis, T. D. Hatchard and M. N. Obrovac, J. Electrochem. Soc., 2012, 159, A1801.

16 Y. Liu, N. Zhang, L. Jiao, Z. Tao and J. Chen, Adv. Funct. Mater., 2015, 25, 214.

17 Z. Li, J. Ding and D. Mitlin, Acc. Chem. Res., 2015, 48, 1657. 18 B. Zhang, G. Rousse, D. Foix, R. Dugas, D. A. D. Corte and J.-M. Tarascon, Adv. Mater., 2016, 28, 9824.

19 A. Darwiche, C. Marino, M. T. Sougrati, B. Fraisse, L. Stievano and L. Monconduit, J. Am. Chem. Soc., 2012, 134, 20805.

20 M. He, K. Kravchyk, M. Walter and M. V. Kovalenko, Nano Lett., 2014, 14, 1255.

21 L. Hu, X. Zhu, Y. Du, Y. Li, X. Zhou and J. Bao, Chem. Mater., 2015, 27, 8138.

22 L. Baggetto, J. K. Keum, J. F. Browning and G. M. Veith, Electrochem. Commun., 2013, 34, 41.

23 Z. Li, X. Tan, P. Li, P. Kalisvaart, M. T. Janish, W. M. Mook, E. J. Luber, K. L. Jungjohann, C. B. Carter and D. Mitlin, Nano Lett., 2015, 15, 6339.

24 S. H. Choi and Y. C. Kang, Nano Res., 2015, 8, 1595.

25 L. Wu, H. Lu, L. Xiao, J. Qian, X. Ai, H. Yang and Y. Cao, J. Mater. Chem. A, 2014, 2, 16424.

26 Y. Jiang, M. Hu, D. Zhang, T. Yuan, W. Sun, B. Xu and M. Yan, Nano Energy, 2014, 5, 60.

27 Y. Liu, N. Zhang, C. Yu, L. Jiao and J. Chen, Nano Lett., 2016, 16, 3321.

28 K. He, F. Lin, Y. Zhu, X. Yu, J. Li, R. Lin, D. Nordlund, T.-C. Weng, R. M. Richards, X.-Q. Yang, M. M. Doeff, E. A. Stach, Y. Mo, H. L. Xin and D. Su, Nano Lett., 2015, 15, 5755.

29 Y. Denis, P. V. Prikhodchenko, C. W. Mason, S. K. Batabyal, J. Gun, S. Sladkevich, A. G. Medvedev and O. Lev, Nat. Commun., 2013, 4, 2922.

30 T. Zhou, W. K. Pang, C. Zhang, J. Yang, Z. Chen, H. K. Liu and Z. Guo, ACS Nano, 2014, 8, 8323.

31 L. David, R. Bhandavat and G. Singh, ACS Nano, 2014, 8, 1759.

32 Y. L. Ding, P. Kopold, K. Hahn, P. A. van Aken, J. Maier and Y. Yu, Adv. Mater., 2016, 28, 7774.
33 Y. Xiao, S. H. Lee and Y.-K. Sun, Adv. Energy Mater., 2017, 7, 1601329.

34 Z. Hu, Q. Liu, S.-L. Chou and S.-X. Dou, Adv. Mater., 2017, 29, 1700606.

35 P. Ge, C. Zhang, H. Hou, B. Wu, L. Zhou, S. Li, T. Wu, J. Hu, L. Mai and X. Ji, Nano Energy, 2018, 48, 617.

36 M. Wan, R. Zeng, K. Chen, G. Liu, W. Chen, L. Wang, N. Zhang, L. Xue, W. Zhang and Y. Huang, Energy Storage Materials, 2018, 10, 114.

37 P. Ge, H. Hou, X. Ji, Z. Huang, S. Li and L. Huang, Mater. Chem. Phys., 2018, 203, 185.

38 B. Jache and P. Adelhelm, Angew. Chem., Int. Ed., 2014, 53, 10169.

39 A. P. Cohn, K. Share, R. Carter, L. Oakes and C. L. Pint, Nano Lett., 2016, 16, 543.

40 H. Kim, J. Hong, G. Yoon, H. Kim, K.-Y. Park, M.-S. Park, W.-S. Yoon and K. Kang, Energy Environ. Sci., 2015, 8, 2963.

41 S.-H. Yu, S. H. Lee, D. J. Lee, Y.-E. Sung and T. Hyeon, Small, 2016, 16, 2146.

42 M. A. Lowe, J. Gao and H. D. Abruña, J. Mater. Chem., 2013, 1, 2094.

43 F. Klein, B. Jache, A. Bhide and P. Adelhelm, Phys. Chem. Chem. Phys., 2013, 15, 15876.

44 Y.-M. Lin, P. R. Abel, A. Gupta, J. B. Goodenough, A. Heller and C. B. Mullins, ACS Appl. Mater. Interfaces, 2013, 5, 8273.

45 P. K. Dutta, U. K. Sen and S. Mitra, RSC Adv., 2014, 4, 43155.

46 Y. Zhu, X. Han, Y. Xu, Y. Liu, S. Zheng, K. Xu, L. Hu and C. Wang, ACS Nano, 2013, 7, 6378.

47 Z. Jian, B. Zhao, P. Liu, F. Li, M. Zheng, M. Chen, Y. Shi and H. Zhou, Chem. Commun., 2014, 50, 1215.

48 J. W. Wang, X. H. Liu, S. X. Mao and J. Y. Huang, Nano Lett., 2012, 12, 5897.

49 C. Ma, J. Xu, J. Alvarado, B. Qu, J. Somerville, J. Y. Lee and Y. S. Meng, Chem. Mater., 2015, 27, 5633.

50 I. A. Courtney and J. Dahn, J. Electrochem. Soc., 1997, 144, 2045.

51 M. Gu, A. Kushima, Y. Shao, J.-G. Zhang, J. Liu, N. D. Browning, J. Li and C. Wang, Nano Lett., 2013, 13, 5203. 